\title{
The nature of the lead-iodine bond in PbI2: A case study for the modelling of lead halide perovskites
}

Costanza Borghesi, ${ }^{\text {ab }}$ Eros Radicchi, ${ }^{\text {ab }}$ Leonardo Belpassi, ${ }^{\text {b }}$ Daniele Meggiolaro, ${ }^{\text {bc }}$ Filippo De Angelis, ${ }^{\text {abc }}$ Francesca Nunzi ${ }^{\text {ab }}$

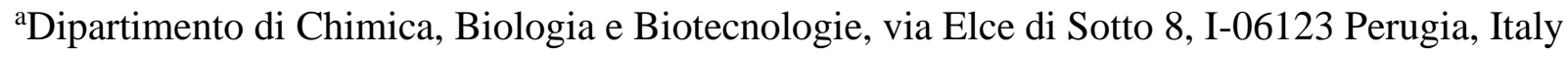

${ }^{\mathrm{b} C}$ Computational Laboratory for Hybrid/Organic Photovoltaics (CLHYO), CNR - ISTM, via Elce di Sotto 8, I-06123 Perugia, Italy

${ }^{\mathrm{c} C o m p u N e t, ~ I s t i t u t o ~ I t a l i a n o ~ d i ~ T e c n o l o g i a, ~ V i a ~ M o r e g o ~ 30, ~} 16163$ Genova, Italy

\begin{abstract}
A detailed knowledge of the basic electronic interactions in lead halide perovskites components ( $\mathrm{PbI}_{2}$ and methylammonium iodide) can possibly drive enhanced solar cell efficiency. We report an extensive investigation on the electronic structure and nature of the chemical bond in the $\mathrm{PbI}_{2}$ perovskite precursor, both in gas and solid state, together with a comparison with available experimental data, which allows to effectively calibrate the computational framework, along with gaining basic understanding on the nature of the $\mathrm{Pb}$ _ I chemical bond. Inclusion of spin orbit coupling and calibrated HF exchange contribution to the DFT hybrid functional are proved essential for an accurate description of the electronic structure of both molecular and solid state $\mathrm{PbI}_{2}$. Such computational framework, calibrated on the model $\mathrm{PbI}_{2}$ system, can be directly translated to the accurate description of the electronic band structure of the prototypical methylammonium lead-iodide perovskite, setting the basis for the trustful modelling of different lead-halide perovskites.
\end{abstract}

\section{Graphical abstract}

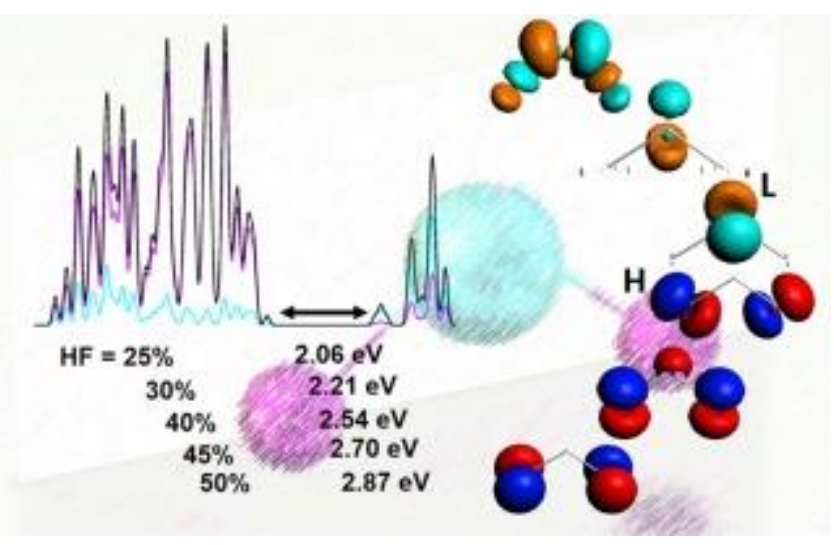

\section{Introduction}

Metal halide perovskites are among the most promising materials for solar cells and optoelectronic devices in general [1], [2], [3], thanks to their fascinating electronic and optical properties, such as direct and tuneable band gap and high optical absorption coefficients, ambipolar charge transport properties and limited charge recombination and scattering at defects. Their general chemical formula is $\mathrm{AMX}_{3}$, where $\mathrm{A}$ is an organic cation, $\mathrm{M}$ is a divalent metal, such as $\mathrm{Pb}, \mathrm{Sn}$, and $\mathrm{X}$ is a halide such as $\mathrm{Cl}, \mathrm{Br}$ and $\mathrm{I}$. The crystal structure is based on $\left[\mathrm{MX}_{6}\right]^{4-}$ metal halide octahedra surrounded by eight 
A-site cations in a (distorted) cubic arrangement. It is worth to recall that lead iodide exists in several polytypes, all of which involve I-Pb-I sandwiches stacked in a variety of ways [4]. Methylammonium lead halide $\left(\mathrm{MAPbX}_{3}\right)$ perovskites are usually employed in photovoltaic applications and can be efficiently synthesized by cheap and easy solution-based methods [5], [6], [7], [8], starting from common materials, namely $\mathrm{PbI}_{2}$ and methylammonium iodide $\left(\mathrm{CH}_{3} \mathrm{NH}_{3} \mathrm{I}\right)$ in an appropriate coordinating solvent, such as dimethylsulfoxide (DMSO), dimethylformamide (DMF) or $\gamma$ butyrolactone (GBL). The perovskite synthesis process relies on the mixing of a metal-halide precursor solution (e.g. a concentrated DMF solution of $\mathrm{PbI}_{2}$ ) with a 1:1 halide salt, containing the A-cation. Upon solvent evaporation, a polycrystalline $\mathrm{MAPbI}_{3}$ film is generated. It is well established that the chemical interactions between the organic and the inorganic components, both in the precursor solution and in the solid state, strongly affect the formation, the optoelectronic properties and the stability of the perovskite materials, that final influence the solar cell efficiency and stability [9].

A detailed knowledge of the basic electronic interactions in the inorganic $\mathrm{PbI}_{2}$ precursor may allow researchers to gain an exhaustive and confident picture on the nature of the $\mathrm{Pb}$ _I bond which may reveal important information on both the perovskite precursors and shed light on the nature of the final perovskite product. From a different perspective, studying $\mathrm{PbI}_{2}$ (both in gas phase and solid state) may represent a way to calibrate electronic structure methods with respect to various variables (e.g. level of theory, inclusion of relativistic effects, etc.), which may deliver important information about the accuracy of such calculation in both solid $\mathrm{PbI}_{2}$ (as a precursor to the perovskite synthesis) and different lead-halide perovskites.

$\mathrm{PbI}_{2}$ at room temperature is a bright yellow crystalline solid. A plethora of experimental and theoretical works have been devoted to the investigation of this material in the solid state [5], [6], [7], [8], including the spectroscopic characterization of the nature of its valence band [10], [11]. To our knowledge, only few studies have investigated the gas-phase $\mathrm{PbI}_{2}$ molecule [6], [12], [13], since this system is difficult to be isolated at room temperature. As a result, the present understanding of the nature of the bond in the $\mathrm{PbI}_{2}$ perovskite precursor is currently lacking, despite its huge popularity among materials for photovoltaic applications. Accordingly, in the present manuscript we report an extensive theoretical investigation of the $\mathrm{PbI}_{2}$ system, both in gas and solid state phase based on Density Functional Theory (DFT). Our aim is twofold: (i) to fine-tune a theoretical framework for the investigation of lead iodide-based systems; and (ii) to get detailed information on the nature of the Pb-I bond which can be generalized to other compounds. In particular, we analyze the structure and electronic properties of the $\mathrm{PbI}_{2}$ molecule, employing various theoretical levels, namely, changing the scalar or fully relativistic Hamiltonian, the DFT functional and the basis set, in order to verify the effect of the employed methodology on the structural and electronic parameters. A comparison with available experimental data allows us to fine-tune the employed theoretical framework. To further characterize the $\mathrm{Pb}-\mathrm{I}$ bond in the $\mathrm{PbI}_{2}$ perovskite precursor, the charge displacement (CD) analysis has been employed [14], highlighting the balance between covalent and ionic character in $\mathrm{PbI}_{2}$. We then move to consider a comparison of the calculated and experimental valence band of $\mathrm{PbI}_{2}$ in the solid state, finding also in this case a primary role of spin orbit coupling (SOC) and of the exact Hartree Fock (HF) exchange contribution in the DFT functional to gain a reliable description of the $\mathrm{PbI}_{2}$ electronic structure. Finally, calculations on the $\mathrm{MAPbI}_{3}$ perovskite material prove that the fined tuned computational protocol can be confidently extended to the investigation of the perovskite materials. 


\section{Computational details}

DFT calculations on the $\mathrm{PbI}_{2}$ molecule have been carried out by using the ADF program [15], [16], [17], designed to perform electronic structure calculations on molecular systems. The one electron equations, arising in the Kohn-Sham formulation of DFT, are solved by SelfConsistent Field calculations. Slater-type orbital basis sets are employed, namely TZ2P and QZ4P, together with three level of frozen core: small $[\mathrm{Pb}(4 \mathrm{~d})-\mathrm{I}(4 \mathrm{p})]$, medium, $[\mathrm{Pb}(4 \mathrm{f})-\mathrm{I}(4 \mathrm{~d})]$, and large $[\mathrm{Pb}(5 \mathrm{~d})-\mathrm{I}(4 \mathrm{~d})]$. The relativistic effects have been included through the ZORA Hamiltonian both in the scalar and fully relativistic form, with the inclusion of the SOC effect [18], [19], [20]. We considered two generalized gradient approximation (GGA) DFT functionals, namely PBE [21] and BLYP [22], [23], [24], and two hybrid DFT functionals, namely B3LYP [25], [26] and PBE0 [27], [28]. In addition, we also carried out selected calculations by employing the PBE0 functional with an exact HF exchange energy percentage increased from 25 to $45 \%$.

The $\mathrm{PbI}_{2}$ photoelectron spectra have been simulated by interpolating the 14 highest occupied molecular orbitals (HOMOs) energy levels computed at various level of theory with Gaussian convolutions (width equal to $0.2 \mathrm{eV}$, arbitrary intensity, corresponding to the MO occupation number).

The electron density change occurring upon formation of an adduct can be analysed by employing the CD function [14], defined as: $\Delta \mathrm{qz}=\int-\infty+\infty \mathrm{dx} \int-\infty+\infty \mathrm{dy} \int-\infty \mathrm{z} \Delta \rho \mathrm{x}, \mathrm{y}, \mathrm{z}^{\prime} \mathrm{dz}$ 'where $\Delta \rho$ is the difference between the electron density of the adduct and the non-interacting fragments placed in the same position they have in the adduct. Accordingly, integrating $\Delta \rho$ along a given $z$ direction (usually the bond axis), we gain at each point of $z$, the electron density transferred from one side to the other side of the perpendicular plane through $z$. A positive (negative) value corresponds to electrons flowing in the direction of decreasing (increasing) $z$. The $\mathrm{CD}$ analysis was successfully employed in the description of both coordination bonds and weak secondary interactions, such as hydrogen and halogen bond [29], [30]. A numerical value of the charge transfer (CT) can be estimated by considering the $\mathrm{CD}$ function value at a specific point between the fragments along the $z$ axis. An adequate choice is the isodensity boundary, where the electron density of the non-interacting fragments become equal. According to the $\mathrm{CD}$ function definition, we selected as constituting fragments the $\mathrm{PbI}+$ and $\mathrm{I}$ - ions in the $\mathrm{PbI}_{2}$ geometry optimized at the selected fully relativistic ZORA Hamiltonian B3LYP/QZ4P level of theory and we rotated the $\mathrm{PbI}_{2}$ molecule so that the bond between the $\mathrm{Pb}$ center and the I- anion lies along the $z$ axis.

To corroborate the observations gathered from the $\mathrm{CD}$ analysis, we also analysed the bonding interactions between $\mathrm{PbI}+$ and I- ions fragments forming the $\mathrm{PbI}_{2}$ complex with the Energy Decomposition Analysis (EDA) scheme, as implemented in the ADF package (see Supporting

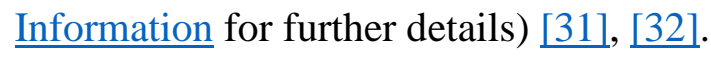

DFT calculations on the solid state $\mathrm{PbI}_{2}$ and $\mathrm{MAPbI}_{3}$ were carried out with the Quantum Espresso package [33]. For $\mathrm{PbI}_{2}$ we modeled a $2 \times 2 \times 2$ supercell with experimental cell parameters of $\mathrm{a}=\mathrm{b}=4.557 \AA$ and $\mathrm{c}=6.979 \AA[34]$, while for $\mathrm{MAPbI}_{3}$ we considered the tetragonal unit cell, made by $4 \mathrm{MAPbI}_{3}$ units and with cell parameters taken from Stoumpos et al. $[35](\mathrm{a}=\mathrm{b}=8.849 \AA$ and $\mathrm{c}=12.642 \AA$ ). The starting geometries were optimized with the PBE functional [21], by using ultrasoft pseudopotentials (for $\mathrm{Pb} 5 \mathrm{~d}$ semi-core states were included in the pseudopotential, i.e. 14 electrons), a cutoff on the wavefunctions of 40 Ryd (320 Ryd on the charge density), and $4 \times 4 \times 2 \mathrm{k}$ points grids in the Brillouin zone. The introduction of dispersion corrections through the DFT-D3 scheme of Grimme [36] only slightly affected the equilibrium geometries found at the PBE level (at fixed cell parameters), with negligible impact on the calculated electronic structures. Single point 
hybrid functional calculations were carried out on the PBE relaxed structures by using the HSE06 functional [37] and including SOC. Norm conserving pseudopotentials were used (for $\mathrm{Pb} 5 \mathrm{~s}, 5 \mathrm{p}$ and $5 \mathrm{~d}$ semi-core electrons have been included in the pseudopotentials, i.e. 22 electrons) [38] with a cutoff on the wavefunctions of 40 Ryd ( 80 Ryd on the Fock exchange grid) and $2 \times 2 \times 2$ grids of k-points in the Brillouin zone for both $\mathrm{PbI}_{2}$ and $\mathrm{MAPbI}_{3}$. The HSE06 functional [37] was employed by considering an exact $\mathrm{HF}$ exchange energy percentage increased up to $50 \%$ for $\mathrm{PbI}_{2}$, while a fixed value of $43 \%$ has been used for $\mathrm{MAPbI}_{3}$. Such value accurately describe the electronic structure of $\mathrm{MAPbI}_{3}$ providing results in good agreement with state of the art GW calculations [39].

\section{Results and discussion}

\subsection{Gas phase $\mathrm{PbI}_{2}$}

Geometry optimizations were carried out on the gas-phase $\mathrm{PbI}_{2}$ molecule, by tuning the theoretical framework, specifically the relativistic ZORA Hamiltonian (scalar or fully relativistic), the functional (GGA or hybrid), the basis set size (TZ2P or QZ4P) and its frozen core (small, medium, large, none). As shown in Tables S1 and S2 inSupporting Information, only minor differences are found by changing the theoretical framework and the DFT computed data are in fairly good agreement with both previous calculations [12], [40] and experimental data [41], [42], [43], with Pb_I bond distances and angles in the range $2.81-2.90 \AA$ and $101-105^{\circ}$, respectively. We then analysed the electron density change occurring upon formation of the $\mathrm{PbI}_{2}$ complex from the $\mathrm{PbI}^{+}$and $\mathrm{I}^{-}$constituting fragments on a selected optimized geometry at the B3LYP (all electron) QZ4P basis set fully relativistic ZORA Hamiltonian level of theory and verifying the effect of different DFT functionals (GGA or hybrid) on the CD analysis. The results of the $\mathrm{CD}$ analysis are reported in Fig. 1 and Tables S3 and S4 in Supporting Information. As shown in Fig. 1a, the employed functionals return the same qualitative picture: $\Delta q$ is positive everywhere in the molecular region, suggesting that, at each point along the internuclear axis $(z)$, there has been a net shift of charge from I- to PbI+ and proving the occurrence of a partial covalent character in the $\mathrm{PbI}_{2}$ system, as confirmed by the isodensity deformation 3D plot, shown in the background. A reasonable estimation of the CT is gained from the $\Delta \mathrm{q}$ value at the isodensity boundary [see Computational Details, Fig. 1a and Table

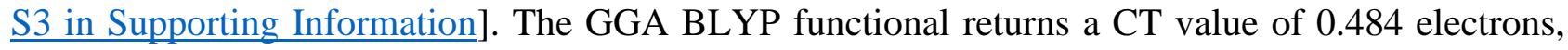
suggesting the presence of a partial covalent character in the $\mathrm{Pb}-\mathrm{I}$ bond, due to a sizeable electron accumulation in the internuclear region. With the other functionals, the computed transferred charge decreases, passing to $0.482 \mathrm{e}$ with $\mathrm{PBE}$ and to $0.462 / 0.454 \mathrm{e}$ with the B3LYP/PBE0 hybrid functionals. By including an increased fraction of exact HF exchange of $30 / 40 / 45 \%$ in the conventional PBE0 functional, a further CT decrease is attained, with computed values of $0.449 / 0.440 / 0.435$ e, respectively. 

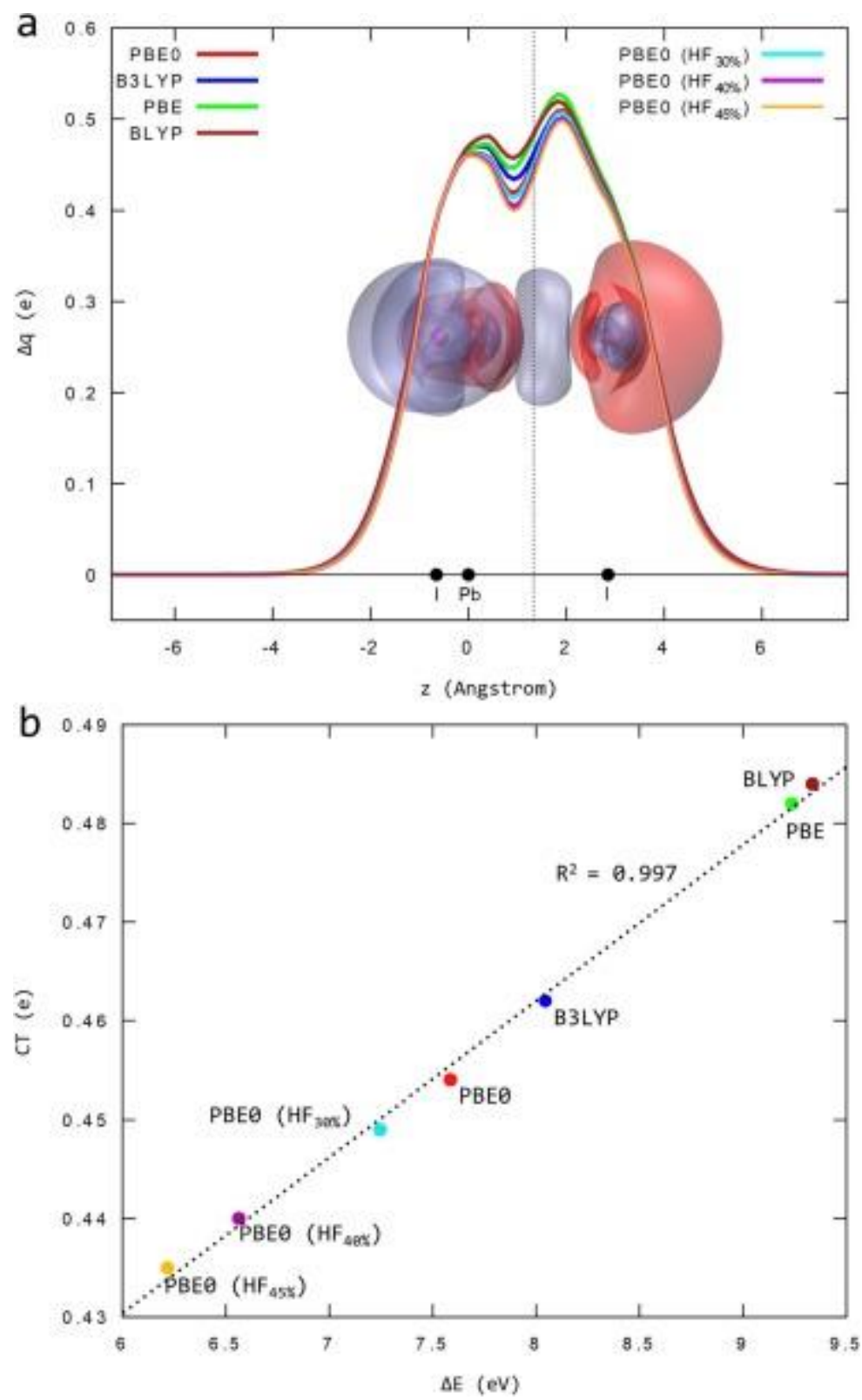

Fig. 1. (a) CD curves ( $\Delta \mathrm{q}$, e, vs $\mathrm{z}, \AA$ ) for the $\mathrm{PbI}_{2}$ molecule (optimized B3LYP/QZ4P geometry) computed with various functionals. The background shows the $3 \mathrm{D}$ isodensity plot of the electron density change occurring upon adduct formation $\left(\Delta \rho= \pm 1.2 \cdot 10^{-6} \mathrm{e} / \mathrm{bohr}^{3}\right.$, negative/positive values in red/grey). The dots on the solid black line represent the nuclei in the $z$ coordinates. The vertical dashed line identifies a conventional boundary between the $\mathrm{PbI}^{+}$and $\mathrm{I}^{-}$fragments. (b) Correlation between the computed $\mathrm{CT}$ (e) and the $\mathrm{I}^{-} \mathrm{HOMO}$ and $\mathrm{PbI}^{+}$LUMO energy difference $(\Delta \mathrm{E}, \mathrm{eV})$. (For interpretation of the references to colour in this figure legend, the reader is referred to the web version of this article.)

Based on this analysis, we conclude that, while the employed functional does not qualitatively affect the characterization of the bond (as covalent), variations in the computed CT values are retrieved that can be related to the electronic structure of the involved fragments. Specifically, we considered the energy difference, $\triangle \mathrm{E}$, between the $\mathrm{I}^{-} \mathrm{HOMO}$ and $\mathrm{PbI}^{+}$LUMO energy levels (see Table $\mathrm{S} 3$ in Supporting Information), which entity should be related to the driving force for the injection of the electron charge from $\mathrm{I}^{-}$to $\mathrm{PbI}^{+}$fragments and, consequently, to the computed CT. As illustrated in Fig. 1 b, a linear correlation between the $\Delta \mathrm{E}$ and $\mathrm{CT}$ values is found, that can be rationalized analysing the energy levels of the $\mathrm{I}^{-}$and $\mathrm{PbI}^{+}$fragments computed with the various functionals. In 
particular, passing from the GGA to hybrid functionals, the $\Delta \mathrm{E}$ decrease is related to a decrease in energy of the $\mathrm{I}^{-} \mathrm{HOMO}$ and an increase in energy of the $\mathrm{PbI}^{+}$LUMO (see Table S3 in Supporting Information) and points at a decrease of the $\mathrm{Pb}-\mathrm{I}$ covalent character. We also apply the EDA [31], [32] as implemented in the ADF program, allowing to identify the various contributions to the chemical bond (see Supporting Information for further details). In particular, we found that the absolute value of the computed orbital interaction term, $\Delta$ Eorb (see Table S4 in Supporting Information), accounting for both polarization and CT contributions, nicely shows the same trend of the CT values, namely, $\triangle$ Eorb decreases with the increasing of the exact HF energy percentage.

We further analysed the effect of the employed functional on the description of the $\mathrm{PbI}_{2}$ electronic structure. We first compared experimental data from photoelectron spectroscopy measurements [10], [13] with the energy levels computed at the fully relativistic ZORA Hamiltonian PBE0/QZ4P level of theory. Specifically, as shown in Table 1, to facilitate comparison on a relative scale, we set to zero the HOMO and the higher lying experimental peak (4a1, [13] A [10]) and we compared the energy differences with the 13 HOMOs energy levels or peaks. The innermost MOs (HOMO-13; HOMO-12) were computed at an average value of $19.21 \mathrm{eV}$, in excellent agreement with the $\left(5 d_{3 / 2}\right)$ innermost peak values, as gained from photoelectron spectra $(20.30 \mathrm{eV}[13]$ and $20.0 \mathrm{eV}[10])$. Similarly, the energy difference between the outermost (HOMO) and innermost minus one (HOMO-9:HOMO-11) energy levels is computed at $16.58 \mathrm{eV}$, in very good agreement with the corresponding $5 \mathrm{~d}_{5 / 2}$ peak values of $17.58[13]$ and $17.5 \mathrm{eV}[10]$ from experiments. Moving to higher energy, the (HOMO-7; HOMO-8) energy levels computed at an average value of $11.92 \mathrm{eV}$ fairly corresponds to the $\mathrm{F}$ peak at $11.4 \mathrm{eV}$ in the photoemission spectrum of Margaritondo et al., [10] while the (HOMO-6) energy level at $7.21 \mathrm{eV}$ corresponds to the $2 \mathrm{a}_{1}(7.42 \mathrm{eV})[13]$ and E $(7.4 \mathrm{eV})[10]$ peaks. Finally, regarding the valence group orbitals, we computed 3 non degenerate energy levels, at $2.05 \mathrm{eV}$ (HOMO-5), $1.33 \mathrm{eV}$ (HOMO-4; HOMO-3) and $0.40 \mathrm{eV}$ (HOMO-2; HOMO-1), that are in good agreement with the data gained from experimental spectra [10], [13]. 
Table 1. Energy values for the computed energy levels and experimental peaks [10], [13] scaled for the outermost energy level (HOMO) or peak $\left(4 \mathrm{a}_{1} / \mathrm{A}\right)$. For almost degenerate computed energy levels an averaged value is supplied.

\begin{tabular}{lllllll} 
PBE0/QZ4P & \multicolumn{3}{c}{ EXP [13] } & \multicolumn{3}{c}{ EXP [10] } \\
HOMO & 0 & $4 \mathrm{a} 1$ & 0 & $\mathrm{~A}$ & $0 \pm 0.4$ \\
HOMO-1; HOMO-2 & 0.40 & $3 \mathrm{~b} 2+1 \mathrm{a} 2$ & $0.3,0.59$ & $\mathrm{~B}$ & $1.2 \pm 0.3$ \\
HOMO-3; HOMO-4 & 1.33 & $1 \mathrm{~b} 1+3 \mathrm{a} 1$ & $1.3,1.42$ & $\mathrm{C}$ & $2.4 \pm 0.4$ \\
HOMO-5 & 2.05 & $2 \mathrm{~b} 2$ & 2.01 & $\mathrm{D}$ & $3.0 \pm 0.4$ \\
HOMO-6 & 7.21 & $2 \mathrm{a} 1$ & 7.42 & $\mathrm{E}$ & $7.4 \pm 0.5$ \\
HOMO-7; HOMO-8 & 11.92 & $/$ & & & $\mathrm{F}$ & $11.4 \pm 0.4$ \\
HOMO-9: HOMO-11 & 16.58 & $5 \mathrm{~d}_{5 / 2}$ & 17.58 & $5 \mathrm{~d}_{5 / 2}$ & $17.5 \pm 0.2$ \\
HOMO-12; HOMO-13 & 19.21 & $5 \mathrm{~d}_{3 / 2}$ & 20.30 & $5 \mathrm{~d}_{3 / 2}$ & $20.0 \pm 0.2$
\end{tabular}

To visualize the comparison between theory and experiment, we report in Fig. 2 the $\mathrm{PbI}_{2}$ experimental HeII $\alpha$ photoelectron spectrum [13] (energy and shape of the bands) together with a Gaussian convolution of the calculated energy levels, following the same scaling procedure outlined above. We note that the innermost peak assigned to the $5 \mathrm{~d} 3 / 2$ orbitals $(29.20 \mathrm{eV})[13]$ is missing in the experimental spectrum drawing [13]. From the comparison in Fig. 2, we figure out a fairly good agreement with the experimental spectrum. As discussed above, the peak computed at ca. $11 \mathrm{eV}$ below the $\mathrm{HOMO}$ is present in the layered $\mathrm{PbI}_{2}$ spectrum, while is completely absent in the gas phase one [10], [13]. Moreover, in the gas phase $\mathrm{PbI}_{2}$ spectrum [13] the high intensity band centred at $14.5 \mathrm{eV}$ can be attributed to species other than $\mathrm{PbI}_{2}$, since it is lacking not only in the simulated, but also in the photoemission spectra from Margaritondo et al. [10]. The data reported in Table 1 and Fig. $\underline{2}$ support the reliability of the employed computational framework for the analysis of the $\mathrm{PbI}_{2}$ electronic structure. 


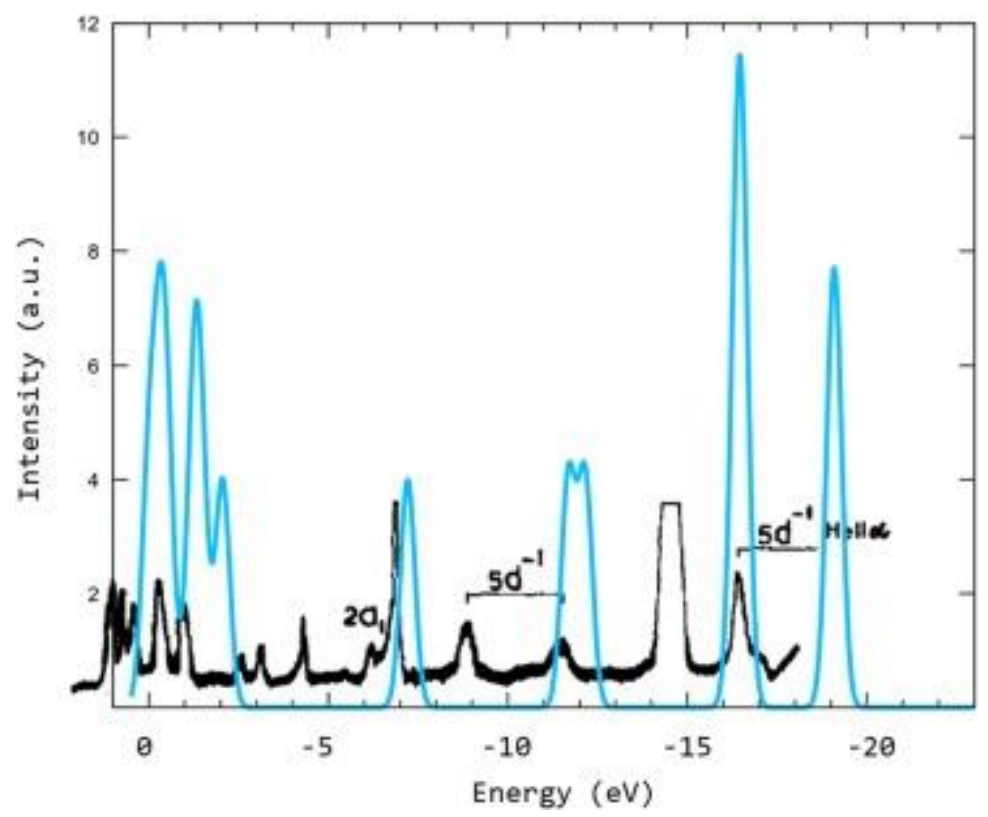

Fig. 2. Full relativistic ZORA Hamiltonian PBE0/QZ4P PbI 2 simulated valence band structure (blue) compared with the experimental HeII $\alpha$ photoelectron spectrum (black line) in gas phase adapted from Ref. [12]. (For interpretation of the references to colour in this figure legend, the reader is referred to the web version of this article.)

We now move to compare the simulated photoelectron spectra computed with the four different employed DFT functionals (see Fig. S1 in Supporting Information). To this aim, we first aligned the HOMO energy level to zero and afterwards we overlapped the four spectra by matching the two innermost peaks of similar shape, while keeping the same energy range $(20 \mathrm{eV})$. The GGA (BLYP and PBE) functionals return overlapping spectra, while the spectra computed with hybrid functionals are slight blue-shifted with respect to the GGA ones, with the one related to PBE0 being at higher energy with respect to the one related to B3LYP, especially for the peaks related to the valence MOs. In order to clarify the nature of the observed differences in the electronic structure description, we repeated the calculations at a selected fixed geometry (B3LYP/QZ4P) and we compared the obtained simulated spectra with the gas phase experimental spectrum from Novak and Potts [13]. The results are reported in Fig. 3a and clearly show that, analogously to the case in Fig. S1, a monotonous shift at higher energy is attained, passing from GGA to hybrid functionals, meaning that the employed functional effectively conditions the computed energy levels. Moreover, the comparison with the experimental spectrum [13] highlights that a better agreement is attained with the spectra computed with the hybrid rather than GGA functionals and that PBE0, entailing a $25 \%$ of exact HF exchange energy contribution, describes the spectrum valence region better than B3LYP (HF 20\%). Accordingly, we verified the effect of the HF exchange contribution on the energy levels calculation by further increasing its percentage, up to an amount of $45 \%$. The results are reported in Fig. $3 \mathrm{~b}$ and clearly show that the valence shell region is in better agreement with the experimental one [13] by employing the PBE0 functional with an exact HF exchange percentage between 40 and $45 \%$. It is also interesting to verify the calculation of the $\mathrm{PbI}_{2} \mathrm{HOMO}-\mathrm{LUMO}$ energy gap $\left(\Delta \mathrm{E}_{\mathrm{H}-\mathrm{L}}\right)$ by varying the DFT functional (fully relativistic B3LYP/QZ4P geometry). As shown below in Table 2, an energy gap of 1.925 and $2.019 \mathrm{eV}$ has been computed with the BLYP and PBE functionals, respectively. By employing the hybrid functionals, the energy gap undergoes a significant widening, reaching 3.144 and even $3.566 \mathrm{eV}$ for B3LYP and PBE0 functionals, respectively. By increasing the HF exchange percentage up to $45 \%$, the energy gap further increases, reaching even a value of $4.830 \mathrm{eV}$. Such an 
increase is related to a sizable stabilization of the HOMO and destabilization of the LUMO, because of the presence of the exact HF exchange in the exchange-correlation energy functional.
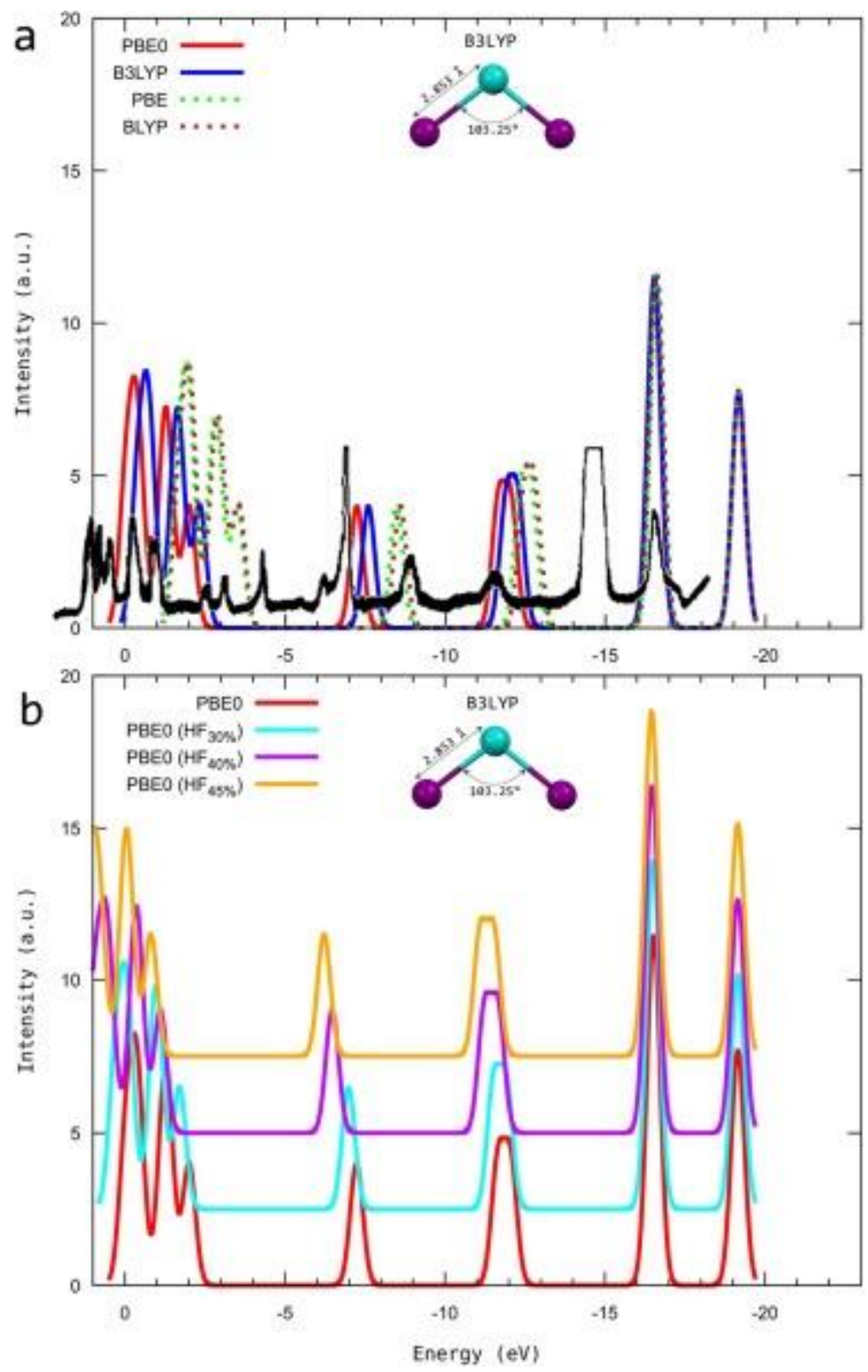

Fig. 3. $\mathrm{PbI}_{2}$ photoelectron spectra computed on the fixed (fully relativistic) B3LYP/QZ4P geometry with: (a) the GGA and hybrid conventional functionals; (b) the hybrid PBE0 with increasing HF exchange percentage, compared to the gas-phase experimental spectrum (solid black line) adapted from reference [13]. 
Table 2. HOMO-LUMO energy gap $\left(\Delta \mathrm{E}_{\mathrm{H}-\mathrm{L}}\right)$ values for the $\mathrm{PbI}_{2}$ molecule computed at the fully relativistic ZORA QZ4P level on the selected fully relativistic B3LYP/QZ4P geometry and energy band gap $\left(\mathrm{E}_{\mathrm{g}}\right)$ for the $\mathrm{PbI}_{2}$ periodic system (PBE and HSE06 functionals, SOC included).

\begin{tabular}{|c|c|c|c|}
\hline \multicolumn{2}{|l|}{$\Delta E_{H-L}(e V)$} & \multicolumn{2}{|l|}{$E_{g}(e V)$} \\
\hline BLYP & 1.925 & & \\
\hline PBE & 2.019 & PBE & 1.35 \\
\hline B3LYP $\left(\mathrm{HF}_{20 \%}\right)$ & 3.144 & & \\
\hline PBE0 $\left(\mathrm{HF}_{25 \%}\right)$ & 3.566 & HSE06 $\left(\mathrm{HF}_{25 \%}\right)$ & 2.059 \\
\hline PBE0 $\left(\mathrm{HF}_{30 \%}\right)$ & 3.880 & HSE06 $\left(\mathrm{HF}_{30 \%}\right)$ & 2.214 \\
\hline PBE0 $\left(\mathrm{HF}_{40 \%}\right)$ & 4.512 & HSE06 $\left(\mathrm{HF}_{40 \%}\right)$ & 2.536 \\
\hline PBE0 $\left(\mathrm{HF}_{45 \%}\right)$ & 4.830 & HSE06 $\left(\mathrm{HF}_{45 \%}\right)$ & 2.703 \\
\hline & & HSE06 $\left(\mathrm{HF}_{50 \%}\right)$ & 2.873 \\
\hline
\end{tabular}

To complete the characterization of the gaseous $\mathrm{PbI}_{2}$ molecule, we further investigate the nature of the $\mathrm{Pb}-\mathrm{I}$ bond computing the MOs energy levels for the isolated $\mathrm{Pb}$ atom and $\mathrm{Pb}^{2+}$ cation and comparing the simulated photoelectron spectra of $\mathrm{PbI}_{2}$ with that of $\mathrm{Pb}$ and $\mathrm{Pb}^{2+}$ (see Fig. $\underline{\mathrm{S} 4}$ in Supporting Information). The main result of this analysis is that the outermost $\mathrm{PbI}_{2}$ valence MOs involve not only the $\mathrm{Pb} 6 \mathrm{p}$ atomic orbitals, but also some electron density on the iodine centers. Specifically, it highlights the MOs characterizing the Pb-I bond, namely the HOMO-3, HOMO-4, HOMO-5, between -8.83 and $-7.95 \mathrm{eV}$, and HOMO-8 $(-19.1 \mathrm{eV})$, showing a prevalent iodine character, and HOMO-6 $(-14.4 \mathrm{eV})$, showing a prevalent lead character. The related discussion is fully reported in the Supporting Information.

\subsection{Solid state $\mathrm{PbI}_{2}$ and $\mathrm{MAPbI}_{3}$}

We now extend our theoretical investigation to the solid state phase, for both the $\mathrm{PbI}_{2}$ and $\mathrm{MAPbI}_{3}$. It is well known that semi-local functionals, like PBE, do not provide an accurate description of the electronic properties of this class of semiconductors [38], [39], [44]. Interestingly, at the PBE level the calculated band gap of the $\mathrm{MAPbI}_{3}$ perovskite is $\sim 1.60 \mathrm{eV}$, a value that accurately matches the experimental value. However, the introduction of SOC strongly narrows the $\mathrm{MAPbI}_{3}$ perovskite band gap to $\sim 0.6 \mathrm{eV}$, requiring higher levels of theory to recover the experimental band-gap [44]. Notably, also these band edge energies are consistently misaligned by semi-local functionals, with hybrid DFT or GW including SOC improving substantially the description of electronic properties [38]. Interestingly, this behaviour is also noted for the calculation of the HOMO-LUMO energy gap for the gas-phase $\mathrm{PbI}_{2}$ (see Table S6 in Supporting Information). For bulk $\mathrm{PbI}_{2}$ we calculate a band gap at the PBE level of $2.18 \mathrm{eV}$, underestimating the experimental value of $2.49 \mathrm{eV}$ [8]. The band gap decreases to a value of $1.35 \mathrm{eV}$ when SOC is included in the calculation. To accurately describe the electronic structure of these materials in the solid phase the use of hybrid functionals with the inclusion of SOC is strictly needed [38], [39]. Among hybrid functionals, PBE0 and HSE06 are common choices in the simulation of periodic solid state systems, since they provide an improved 
description of the electronic properties of semiconductors, such as band gaps, compared to semi-local functionals [45]. The HSE06 functional is based on a screened coulomb kernel and guarantees a faster convergence with the k-points sampling of the Brillouin zone with respect to the PBE0 functional, where the full coulomb kernel is retained. This faster convergence is crucial in solid state periodic calculations to make hybrid DFT simulations practical. Previous studies demonstrated that the use of HSE06 functional (SOC included) with an increased fraction of HF up to $43 \%$ can provide a good estimate of the electronic band edges in $\mathrm{MAPbI}_{3}$, in agreement with experiments and state of the art GW calculations [38], [39]. In order to investigate the effects of increased HF exchange on the electronic properties of solid $\mathrm{PbI}_{2}$, we calculated the total electronic density of states of this phase by using the HSE06 functional and including SOC for increasing values of HF exchange percentage in the functional. Results have been thus compared with available experimental photoemission data [10].

In Fig. 4a the calculated density of states (DOS) of solid $\mathrm{PbI}_{2}$ in the valence band region for increasing values of the exchange fraction are reported. Exchange fractions from the default value of $25 \%$ up to a value of $50 \%$ have been used. The calculated band gaps of these systems are reported in Table 2 , along with the HOMO-LUMO energy gap $\left(\Delta \mathrm{E}_{\mathrm{H}-\mathrm{L}}\right)$ of the $\mathrm{PbI}_{2}$ molecule evaluated at different levels of theory as discussed before, while the density of states (DOS) projected on atomic orbitals (PDOS) have been compared in Fig. S5 in Supporting Information. As shown in Fig. 4a, by increasing the HF exchange percentage, a monotonous shift at lower energy of the valence states is attained, as previously observed for the molecule in the gas phase. On the other hand, the $\mathrm{PbI}_{2}$ conduction band is gradually shifted to higher energy by increasing the HF exchange percentage (see Fig.

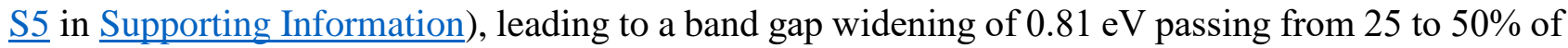
$\mathrm{HF}$ exchange. The analysis of the calculated band gaps of solid $\mathrm{PbI}_{2}$ (see Table 2) highlights that a value of HF exchange between 40 and $45 \%$ in the HSE06 functional can provide values in good agreement with the experimental band gap of $\mathrm{PbI}_{2}$, i.e. $\sim 2.5 \mathrm{eV}$ [7], [8]. The calculated band gaps for such fractions of exchange, 2.54 and $2.70 \mathrm{eV}$, slightly overestimate the experimental value, but it should be noted that these calculations do not include excitonic effects which are in the $\sim 0.1 \mathrm{eV}$ range in $\mathrm{PbI}_{2}$ [46]. The contributions of $\mathrm{Pb}$ and I atoms to the total DOS are slightly affected by the $\mathrm{HF}$ exchange percentage (see Fig. S5 in Supporting Information), but, on overall, confirm that the valence band is mainly constituted by iodine, while the conduction band is dominated by lead. 


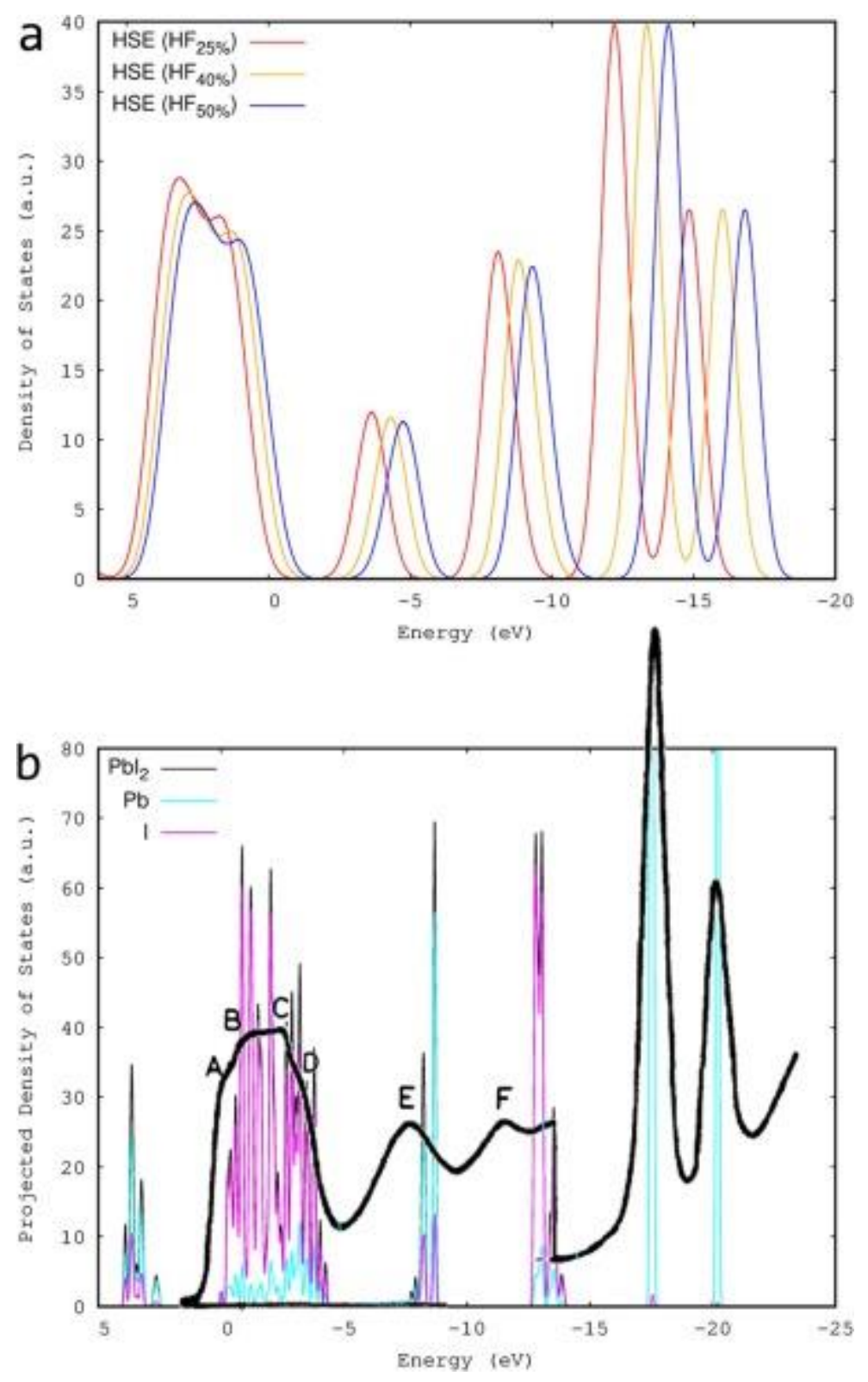

Fig. 4. (a) Total valence density of states of $\mathrm{PbI}_{2}$ calculated with the HSE06 functional (SOC included) with increasing HF exchange percentage; (b) comparison of the calculated DOS at the HSE06-SOC level (HF 40\%) with the experimental photoemission spectrum of $\mathrm{PbI}_{2}$.

In Fig. $4 \mathrm{~b}$ the calculated DOS at the HSE06 level (HF 40\%) of $\mathrm{PbI}_{2}$ are compared with the experimental photoemission spectrum. The calculated DOS with the increased fraction of exchange agrees fairly well with experiments, although some deviations are reported due to the limited accuracy of DFT in the description of photoemission processes in solids, where screening effects between charges play an important role [38], [39], [44].

It is important to note that results obtained by the PBE0 functional for the $\mathrm{PbI}_{2}$ in the gas phase are not directly comparable with results obtained using the HSE06 functional for the solid phase. The latter functional is based on a screened coulomb kernel, thus slightly lower values of HF exchange fractions when using the PBE0 functional in the modelling of solids are expected to provide results comparable with the HSE06 functional.

The optimal fraction of HF exchange in the HSE06 functional found for $\mathrm{PbI}_{2}$ nicely matches the fraction of HF exchange used for the accurate description of the electronic properties of $\mathrm{MAPbI}_{3}$ [39]. 
This suggests that the basic electronic structure properties of $\mathrm{PbI}_{2}$ and $\mathrm{MAPbI}_{3}$ are indeed related. In Fig. 5a and $\mathrm{b}$ the total $\mathrm{PDOS}$ of $\mathrm{PbI}_{2}$ and $\mathrm{MAPbI}_{3}$ perovskite calculated with the HSE06 functional (SOC included) and with HF fractions of $40 \%$ and $43 \%$, respectively, are compared. The top of the valence band has been aligned to zero in both cases. The analysis of the PDOS confirms that $\mathrm{PbI}_{2}$ and $\mathrm{MAPbI}_{3}$ share a globally similar electronic structure, with I and $\mathrm{Pb}$ ions determining valence and conduction band edges, respectively, in the two phases. Small variations in the electronic structures of the two phases can be ascribed to the slightly different fractions of exchange included in the functional in the two cases. To summarize, hybrid functionals with the inclusion of SOC and higher fractions of $\mathrm{HF}$ exchange (up to 45\%) represent a valuable tool to accurately model the electronic structure of layered $\mathrm{PbI}_{2}$ as well as $\mathrm{MAPbI}_{3}$. By tuning the exchange fraction parameter, this method is thus applicable to the study of the electronic properties of other perovskites systems within a unified theoretical framework.

a

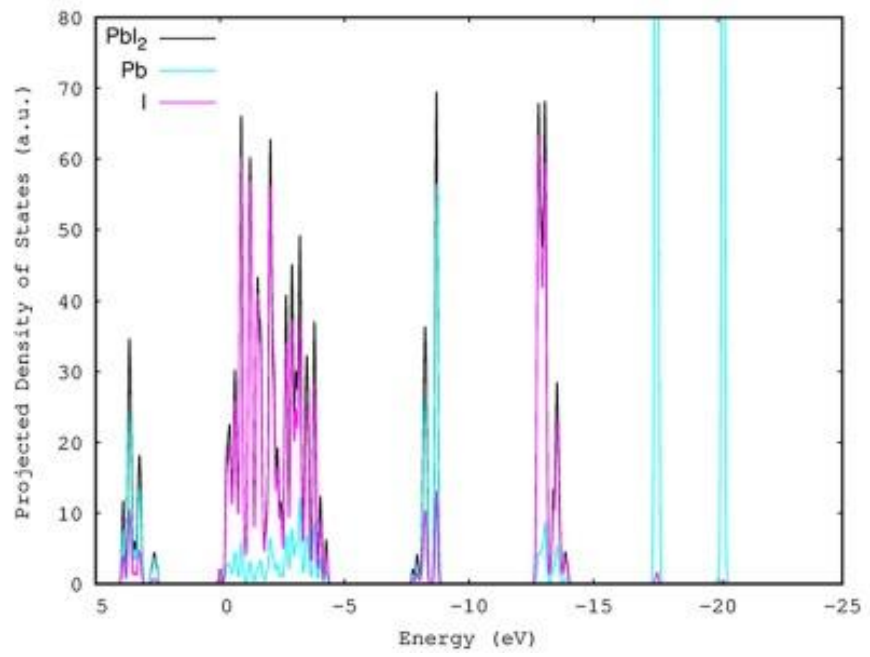

b

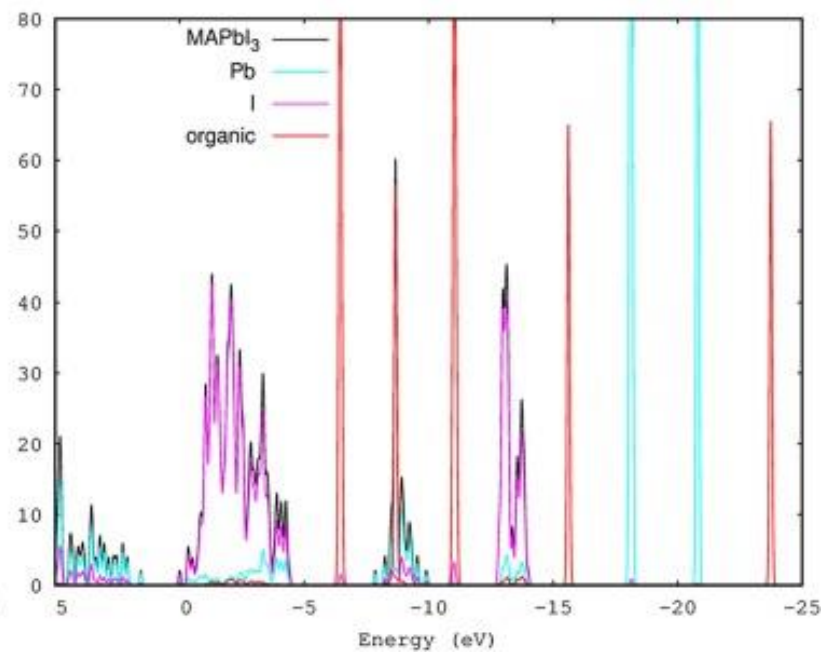

Fig. 5. Comparison between the (a) $\mathrm{PbI}_{2}$ and (b) the $\mathrm{MAPbI}_{3}$ band structures computed with the HSE06 functional with a $40 \%$ and $43 \% \mathrm{HF}$ exchange percentages, respectively. Top of the VBs has been set to zero. Contributions of $\mathrm{Pb}, \mathrm{I}$ and the sum of the organic part $(\mathrm{C}, \mathrm{N}, \mathrm{H})$ to the total $\mathrm{MAPbI}_{3} \mathrm{DOS}_{\mathrm{S}}$ are reported respectively as cyan, magenta and red lines. (For interpretation of the references to colour in this figure legend, the reader is referred to the web version of this article.)

\section{Conclusions}

By means of high level quantum mechanics calculations based on DFT we have investigated the nature of the $\mathrm{Pb}$-I bond in both the gas-phase and solid state $\mathrm{PbI}_{2}$. We fine-tuned the theoretical framework by exploring variations in the Hamiltonian, DFT functional and basis sets and by comparing the obtained results with the available experimental data. Inclusion of SOC in the relativistic effects description and of the HF exchange in the DFT functional are shown to play a key role to get a reliable representation of the $\mathrm{PbI}_{2}$ electronic structure. Specifically, we verified by applying the $\mathrm{CD}$ analysis that the $\mathrm{Pb}$ _ I bond has a partial covalent character, whose entity decreases by increasing the HF percentage in the DFT functional. By employing the PBE0 functional with an increased exact HF percentage (40-45\%), a better agreement of the computed $\mathrm{PbI}_{2}$ photoelectron spectra with the available experimental ones is attained. The analysis of the electronic structure also highlights that, while the $\mathrm{PbI}_{2} \mathrm{HOMOs}$ are essentially localized on the I atoms, some lower-lying HOMOs show a significant $\mathrm{Pb}$ contribution, thus participating to the definition of the $\mathrm{Pb}$ _ I bond character. The analysis of the electronic band structure in the $\mathrm{PbI}_{2}$ solid state phase confirms this picture, showing that the inclusion of the SOC effects and of a sizable HF exchange percentage in the 
hybrid functional play a key role for an accurate description of such system. The optimized computational framework has been applied also to the characterization of the electronic band structure of the lead halide perovskite material, thus proving that the $\mathrm{PbI}_{2}$ and $\mathrm{MAPbI}_{3}$ solid state materials can be treated with the same theoretical approach, setting the basis for the trustful modelling of different lead-halide perovskites.

\section{Acknowledgements}

The authors acknowledge support from the Ministero Istruzione dell'Università e della Ricerca (MIUR) and the University of Perugia through the program "Dipartimenti di Eccellenza 2018-2022" (grant AMIS) and from the European 531 Union's Horizon 2020 research and innovation programme 532 under Grant Agreement No 764047 of the Espresso project.

\section{References}

[1] H.J. Snaith Perovskites: the emergence of a new era for low-cost, high-efficiency solar cells J. Phys. Chem. Lett., 4 (2013), pp. 3623-3630

[2] M.A. Green, A. Ho-Baillie, H.J. Snaith The emergence of perovskite solar cells Nat. Photon., 8 (2014), pp. 506-514

[3] Photovoltaic Research | NREL, Available at: https://www.nrel.gov/pv/.

[4] P.A. Beckmann A review of polytypism in lead iodide Cryst. Res. Technol., 45 (2010), pp. 455460

[5] E.S. Claudio, H.A. Godwin, J.S. Magyar, Fundamental coordination chemistry, environmental chemistry, and biochemistry of lead(II), in: K.D. Karlin (Ed.) Progress in Inorganic Chemistry, Vol 512003, pp. 1-144.

[6] M. Hargittai Molecular structure of metal halides Chem. Rev., 100 (2000), pp. 2233-2301

[7] D.S. Ahlawat Study of band gap energy and thermal properties of $\mathbf{P b I}_{2}$ by photoacoustic spectroscopy Mod. Phys. Lett. B, 26 (2012), p. 1250098

R. Ahuja, H. Arwin, A.F.D. Silva, C. Persson, J.M. OsorioGuillén, J.S.D. Almeida, C.M. Araujo, E. Veje, N. Veissid, C.Y. An, I. Pepe, B. Johansson Electronic and optical properties of lead iodide J. Appl. Phys., 92 (2002), pp. 7219-7224

[9] E. Radicchi, E. Mosconi, F. Elisei, F. Nunzi, F. De Angelis Understanding the solution chemistry of lead halide perovskites precursors ACS Appl. Energy Mater. (2019)

[10] G. Margaritondo, J.E. Rowe, M. Schlüter, G.K. Wertheim, F. Levy, E. Mooser Photoemission spectroscopy of layered halides: $\mathbf{P b I}_{2}$ and BiI3 3 Phys. Rev. B, 16 (1977), pp. 2934-2937

[11] M. Scrocco X-ray and electron-energy-loss spectroscopies of $\mathbf{P b I}_{2}, \mathbf{P b F}_{2}$ and $\mathrm{CdI}_{2} \mathrm{~J}$. Electron. Spectrosc. Relat. Phenom., 48 (1989), pp. 363-374

[12] M. Benavides-Garcia, K. Balasubramanian Bond-energies, ionization-potentials, and the singlet-triplet energy separations $\mathrm{Of} \mathrm{SnCl}_{2}, \mathrm{SnBr}_{2}$, $\mathrm{SnI}_{2}, \mathrm{PbCl}_{2}, \mathrm{PbBr}_{2}, \mathrm{PbI}_{2}$, and their positiveions J. Chem. Phys., 100 (1994), pp. 2821-2830 
[13] I. Novak, A.W. Potts Ultraviolet photoelectron spectra of gas phase and condensed tin and lead dihalides ( $\mathbf{M X}_{2} ; \mathbf{M}=\mathbf{S n}, \mathbf{P b} ; \mathbf{X}=\mathbf{C l}, \mathbf{B r}$, I) J. Electron Spectrosc. Relat. Phenom., 33 (1984), pp. 1-7

[14] L. Belpassi, I. Infante, F. Tarantelli, L. Visscher The chemical bond between Au(I) and the noble gases. Comparative study of $\mathrm{NgAuF}$ and $\mathrm{NgAu}^{+}(\mathrm{Ng}=\mathrm{Ar}, \mathrm{Kr}, \mathrm{Xe})$ by density functional and coupled cluster methods J. Am. Chem. Soc., 130 (2008), pp. 1048-1060

[15] G. te Velde, F.M. Bickelhaupt, E.J. Baerends, C. Fonseca Guerra, S.J.A. van Gisbergen, J.G. Snijders, T. Ziegler Chemistry with ADF J. Comput. Chem., 22 (2001), pp. 931967

[16] ADF 2014, SCM, Theoretical Chemistry, Vrije Universiteit, Amsterdam, The Netherlands, 2014.

[17] C. Fonseca Guerra, J.G. Snijders, G. te Velde, E.J. Baerends Towards an order-N DFT method Theor. Chem. Acc., 99 (1998), pp. 391-403

[18] E. van Lenthe, E.J. Baerends, J.G. Snijders Relativistic regular two-component hamiltonians J. Chem. Phys., 99 (1993), pp. 4597-4610

[19] F. Wang, T. Ziegler, E. van Lenthe, S.J.A. van Gisbergen, E.J. Baerends The calculation of excitation energies based on the relativistic two-component zeroth-order regular Approximation and time-dependent density-functional with full use of symmetry J. Chem. Phys., 122 (2005)

[20] C.A. Gaggioli, L. Belpassi, F. Tarantelli, D. Zuccaccia, J.N. Harvey, P. Belanzoni Dioxygen insertion into the gold(I)-hydride bond: spin orbit coupling effects in the spotlight for oxidative addition Chem. Sci., 7 (2016), pp. 7034-7039

[21] J.P. Perdew, K. Burke, M. Ernzerhof Generalized gradient approximation made simple Phys. Rev. Lett., 77 (1996), pp. 3865-3868

[22] A.D. Becke Density-functional exchange-energy approximation with correct asymptoticbehavior Phys. Rev. A, 38 (1988), pp. 3098-3100

[23] C. Lee, W. Yang, R.G. Parr Development of the Colle-Salvetti correlation-energy formula into a functional of the electron-density Phys. Rev. B, 37 (1988), p. 785

[24] B. Miehlich, A. Savin, H. Stoll, H. Preuss Results obtained with the correlation-energy density functionals of Becke and Lee, Yang and Parr Chem. Phys. Lett., 157 (1989), pp. 200-206

[25] A.D. Becke Density-functional thermochemistry 3. The role of exaxct exchange J. Chem. Phys., 98 (1993), pp. 5648-5652

[26] P.J. Stephens, F.J. Devlin, C.F. Chabalowski, M.J. Frisch Ab-initio calculation of vibrational absorption and circular-dichroism spectra using density-functional force-fields J. Phys. Chem., 98 (1994), pp. 11623-11627

[27] J.P. Perdew, M. Emzerhof, K. Burke Rationale for mixing exact exchange with density functional approximations J. Chem. Phys., 105 (1996), pp. 9982-9985

[28] C. Adamo, V. Barone Toward reliable density functional methods without adjustable parameters: the PBE0 model J. Chem. Phys., 110 (1999), pp. 6158-6170 
[29]

F. Nunzi, D. Cesario, L. Belpassi, F. Tarantelli, L.F. Roncaratti, S. Falcinelli, D. Cappelletti, F. Pira ni Insight into the halogen-bond nature of noble gas-chlorine systems by molecular beam scattering experiments, Ab initio calculations and charge displacement analysis Phys. Chem. Chem. Phys., 21 (2019), pp. 7330-7340

[30] D. Cappelletti, V. Aquilanti, A. Bartocci, F. Nunzi, F. Tarantelli, L. Belpassi, F. Pirani Interaction of $\mathrm{O}_{2}$ with $\mathrm{CH}_{4}, \mathrm{CF}_{4}$, and $\mathrm{CCl}_{4}$ by molecular beam scattering experiments and theoretical calculations J. Phys. Chem. A, 120 (2016), pp. 5197-5207

[31] K. Morokuma Molecular orbital studies of hydrogen bonds. 3. $\mathrm{C}=\mathbf{O} \mathrm{H}-\mathrm{O}$ hydrogen bond in $\mathrm{H}_{2} \mathrm{CO} \ldots \mathrm{H}_{2} \mathrm{O}$ and $\mathrm{H}_{2} \mathrm{CO} \ldots$ 2 $\mathrm{H}_{2} \mathrm{O}$ J. Chem. Phys., 55 (1971), pp. 1236-1244

[32] T. Ziegler, A. Rauk $\mathbf{C O}, \mathbf{C S}, \mathbf{N}_{2}, P_{3}$, and $\mathbf{C N C H}_{3}$ as $\boldsymbol{\sigma}$-donor and $\pi$-acceptors - theoretical study by the Hartree-Fock-Slater transition-state method Inorg. Chem., 18 (1979), pp. 17551759

[33]

P. Giannozzi, S. Baroni, N. Bonini, M. Calandra, R. Car, C. Cavazzoni, D. Ceresoli, G.L. Chiarotti, M. Cococcioni, I. Dabo, et al. QUANTUM ESPRESSO: a modular and open-source software project for quantum simulations of materials J. Phys.: Condes. Matt., 21 (2009)

[34] R.S. Mitchell Structural polytypism of lead iodide and its relationship to screw dislocations Kristall, 111 (1959), pp. 372-384

[35] C.C. Stoumpos, C.D. Malliakas, M.G. Kanatzidis Semiconducting tin and lead iodide perovskites with organic cations: phase transitions, high mobilities, and near-infrared photoluminescent properties Inorg. Chem., 52 (2013), pp. 9019-9038

[36] S. Grimme, J. Antony, S. Ehrlich, H. Krieg A consistent and accurate Ab initio parametrization of density functional dispersion correction (DFT-D) for the 94 elements H-Pu J. Chem. Phys., 132 (2010), p. 154104

[37] J. Heyd, G.E. Scuseria, M. Ernzerhof Hybrid functionals based on a screened coulomb potential J. Chem. Phys., 118 (2003), pp. 8207-8215

[38] D. Meggiolaro, F. De Angelis First-principles modeling of defects in lead halide perovskites: best practices and open issues ACS En. Lett., 3 (2018), pp. 2206-2222

[39] M.-H. Du Density functional calculations of native defects in $\mathrm{CH}_{3} \mathrm{NH}_{3} \mathrm{PbI}_{3}$ : effects of spinorbit coupling and self-interaction error J. Phys. Chem. Lett., 6 (2015), pp. 1461-1466

[40] S.T. Howard Ab-initio effective core potential calculations On $\mathrm{HgI}_{2}, \mathrm{PtI}_{2}$, and PbI2 J. Phys. Chem., 98 (1994), pp. 6110-6113

[41] A. Gershikov, E.Z. Zasorin, A.V. Demidov, V.P. Spiridonov Zh. Struct. Khim., 27 (1986), p. 36

[42] A.V. Demidov, A. Gershikov, E.Z. Zasorin, V.P. Spiridonov, A.A. Ivonov Zh. Struct. Khim., 24 (1983), p. 9

[43] V.I. Bazhanov Zh. Struct. Khim., 32 (1983), p. 54

[44] P. Umari, E. Mosconi, F. De Angelis Relativistic GW calculations on $\mathrm{CH}_{3} \mathrm{NH}_{3} \mathrm{PbI}_{3}$ and $\mathrm{CH}_{3} \mathrm{NH}_{3} \mathrm{SnI}_{3}$ perovskites for solar cell applications Sci. Rep., 4 (2014), p. 4467 
[45] J. Paier, M. Marsman, K. Hummer, G. Kresse, I.C. Gerber, J.G. Ángyán Screened hybrid density functionals applied to solids J. Chem. Phys., 124 (2006), p. 154709

[46] C. Gähwiller, G. Harbeke Excitonic effects in the electroreflectance of lead iodide Phys. Rev., 185 (1969), pp. 1141-1149 